\title{
SÍNTESIS Y CARACTERIZACIÓN DE NANOPARTÍCULAS DE COBRE (NPs Cu) POR EL MÉTODO POLIOL ASISTIDO VÍA MICROONDAS (MW)
}

\author{
Aldo Javier Guzmán Duxtan ${ }^{* a}$, Joel Claudio Rengifo Maravía, \\ Jezabel Milagros Echevarría Muñoz ${ }^{\mathrm{a}}$
}

\begin{abstract}
RESUMEN
En el presente trabajo se describe la síntesis de nanopartículas de cobre (NPs $\mathrm{Cu}$ ) por el método de poliol asistido vía microondas (MW), a partir del precursor acetato de cobre sintetizado, $\mathrm{Cu}(\mathrm{Ac})_{2}$. El precursor se sintetizó usando la técnica Schlenk con calentamiento convencional bajo reflujo en un tiempo mayor a 8 horas. Los reactivos que se utilizaron para preparar las NPs $\mathrm{Cu}$ fueron el Etilenglicol (EG) como solvente, polivinilpirrolidona (PVP) como agente dispersante, ácido ascórbico (AA) y ácido cítrico (AC) como agentes reductores. El proceso de síntesis se realizó en un horno MW adaptado para síntesis química. Se evaluó tres variables para observar la influencia en el tamaño de las nanopartículas, tales como la relación molar $\mathrm{AA} / \mathrm{Cu}(\mathrm{Ac}) 2$, el peso molecular del PVP de $10 \mathrm{KD}, 40 \mathrm{KD}$ y el tipo de procedimiento. Tanto el precursor como las NPs $\mathrm{Cu}$ se caracterizaron con las técnicas UVVis, DRX FTIR-ATR y FRX. Finalmente, se realizó la deconvolución del plasmón superficial característico de las NPs Cu espectro UV- Vis y mediante el análisis factorial se estimó las tendencias del tamaño de grano a partir de la longitud de onda y el ancho de banda de las funciones resultantes de la deconvolución.
\end{abstract}

Palabras clave: Nanopartículas de cobre, acetato de cobre, poliol, microondas, deconvolución, análisis factorial.

\footnotetext{
a Laboratorio de Química Cuántica, Nuevos Materiales e Innovación Tecnológica (LQCNMIT), del Departamento Académico de Fisicoquímica de la FQIQ, UNMSM.

*aldo.guzman@unmsm.edu.pe
} 


\title{
SYNTHESIS AND CHARACTERIZATION OF COPPER NANOPARTICLES (Cu NPs) BY METHOD POLIOL ASSISTED VIA MICROWAVE (MW)
}

\begin{abstract}
This paper describes the synthesis of copper nanoparticles ( $\mathrm{Cu}$ NPs) by the microwaveassisted polyol method (MW) from a synthesized copper acetate precursor, $\mathrm{Cu}(\mathrm{Ac})_{2}$. The precursor was synthesized using the Schlenk with technique conventional heating under reflux in a time greater than 8 hours. The reagents that were used to prepare $\mathrm{Cu}$ NPs were Ethylene glycol (EG) as solvent, polyvinylpyrrolidone (PVP) as dispersing agent, ascorbic acid (AA) and citric acid (AC) as reducing agents. The synthesis process was carried out in an MW furnace adapted for chemical synthesis. Three variables were evaluated to observe the influence on the size of the nanoparticles, such as the $\mathrm{AA} / \mathrm{Cu}(\mathrm{Ac}) 2$ mole ratio, the molecular weight of the PVP 10KD, 40KD and the type of procedure. The Precursor and the $\mathrm{Cu}$ NPs were characterized with UV-Vis, DRX FTIR-ATR and FRX techniques. Finally, the deconvolution of surface plasmon characteristic of the Cu NPs in the UV-Vis spectrum was performed and, by means of factorial analysis, grain size trends were estimated from wavelength and bandwidth of the resulting functions of the deconvolution.
\end{abstract}

Key words: Copper nanoparticles, copper acetate, polyol, microwave, deconvolution, factor analysis.

\section{INTRODUCCIÓN}

Las nanopartículas de cobre (NPs $\mathrm{Cu}$ ) han atraído un gran interés durante los últimos años debido a sus características y excelentes propiedades físicas y químicas que se diferencian de las partículas de mayor tamaño. Las propiedades mejoradas son producto del tamaño nanométrico, la morfología de la superficie y el área superficial, debido al efecto del confinamiento cuántico. En el área de la catálisis heterogénea se utiliza con mayor frecuencia como catalizadores en la electrorremediación de aguas contaminadas y en la desintoxicación de gases ${ }^{1}$. En la electrónica como electrodos, líneas conductoras y tintas de impresión en la microfabricación de conductores ${ }^{2}$. Por otro lado, se han considerado como una alternativa de las nanopartículas de oro (NPs Au), de plata (NPs Ag) y de platino (NPs Pt) ${ }^{3}$, teniendo en cuenta al cobre metálico que es un material más rentable.

El método de poliol se utiliza para sintetizar nanopartículas de diferentes metales consiste de manera general en utilizar un poliol como solvente y agente dispersante, siendo este uno de los métodos más utilizados en la síntesis química de NPs $\mathrm{Cu}$. Yu y su equipo ${ }^{4}$, sintetizaron NPs $\mathrm{Cu}$ con tamaño de partícula entre 5-10nm mediante el método Poliol, utilizando agua y EG como solventes, AA como agente antioxidante y reductor y PVP como controlador 
de tamaño y agente dispersante. Zhang ${ }^{5}$ preparó NPs $\mathrm{Cu}$ estables con un tamaño promedio entre $1,4 \pm 0,6 \mathrm{~nm}$ a $3,1 \pm 0,5 \mathrm{~nm}$ y con una morfología esferoidal sintetizada a temperatura del ambiente, utilizando EG, $\mathrm{NaBH}_{4}$ como agente reductor con un copolímero de etileno en medio alcalino y PVP; determinando que el aumento de la relación Precursor/PVP disminuye el tamaño de las NPs. Chum ${ }^{6}$ a diferencia de los antecedentes anteriores utilizaron como intermediario un complejo $\mathrm{PVP}-\mathrm{Cu}^{+1}$ que luego fue reducido por $\mathrm{NaBH}_{4}$ formando las $\mathrm{NPs} \mathrm{Cu}$ entre 9-33nm que se aplicaron en tintas conductoras. Planteó que la formación del complejo PVP-Cu $\mathrm{Cu}^{+1}$ favoreció la dispersión de las nanopartículas, disminuyendo la velocidad de crecimiento de las NPs $\mathrm{Cu}$.

El método del poliol también se puede utilizar mediante el calentamiento MW. Blosi ${ }^{7}$, planteó la preparación de NPs Cu a partir de acetato de Cu monohidratado, EG, PVP y AA; mediante el calentamiento vía MW, determinó la influencia de los parámetros como la relación molar del agente reductor/precursor, temperatura y el orden de adicción de los reactivos en la optimización del proceso, obteniendo NPsCu de $46 \pm 9 \mathrm{~nm}$. En cambio, Kawasaki y su equipo $^{8}$, sintetizaron nanoclusters $\mathrm{Cu}$ sin la adición de un agente reductor fuerte y agente protector; se realizó a partir de cloruro de cobre, EG y $\mathrm{NaOH}$ con calentamiento vía $\mathrm{MW}$ en atmósfera de nitrógeno. Nikkam9 ${ }^{9}$ utilizó un reactor $\mathrm{MW}$, utilizó acetato de $\mathrm{Cu}$ monohidratado como precursor, EG, PVP y AA, mediante este procedimiento se obtuvieron NPs Cu esféricas con un tamaño de partícula entre $75 \pm 25 \mathrm{~nm}$. De forma similar Nishioka y su equipo ${ }^{10}$, sintetizaron NPs Cu de un tamaño de $63 \mathrm{~nm}$, a partir de acetato de cobre, EG y PVP, llevándolo a cabo en un reactor en flujo con MW. A diferencia del grupo de investigación de Raspolli ${ }^{11}$, que sintetizaron NPs $\mathrm{Cu}$ en ausencia de un agente estabilizante, partieron de acetato de cobre, pero utilizaron al etanol como solvente, AA como agente reductor y $\mathrm{NaOH}$, llevando a cabo la reacción en un reactor MW; las NPs Cu obtenidas tuvieron un menor tamaño $8,7 \pm 3,9 \mathrm{~nm}$. Por tanto, se deduce que, el método de poliol asistido vía MW también permite obtener NPs $\mathrm{Cu}$ con una baja distribución de tamaño de partícula y con una morfología controlada, siendo más eficiente con respecto al calentamiento convencional, ya que se acelera la reacción química, obteniendo el producto en un menor tiempo.

En este trabajo se sintetizó NPs Cu por el método poliol asistido vía MW y se evaluó la influencia del tipo de procedimiento a seguir, la relación molar de agente reductor/precursor de cobre (AA/Cu(Ac)2), y el peso molecular del PVP, para identificar las condiciones óptimas de síntesis mediante la deconvolución de los espectros UV-Vis y el análisis factorial. Las NPs $\mathrm{Cu}$ se caracterizaron mediante las técnicas UV-VIS, DRX y FRX.

\section{PARTE EXPERIMENTAL}

\section{Reactivos}

Ácido acético glacial (Merck Millipore), anhídrido acético (Merck Millipore), $\mathrm{Cu}(\mathrm{Ac})_{2}$ (sintetizado en el laboratorio con $99 \%$ de pureza), EG (Merck Millipore), AA (Sigma Aldrich), AC (Sigma Aldrich), PVP de 10KD y 40KD (Sigma Aldrich), óxido cúprico (sintetizado en el laboratorio con $99 \%$ de pureza). 


\section{Materiales}

Balones de 250mL, tubos Schlenk de 100ml, línea Schlenk para aplicaciones en atmósfera controlada y vacía, filtros Schlenk para atmósfera inerte, columnas refrigerantes de tipo serpentín y Liebig, dos trampas para gases (con silicona líquida) con conexión a sistema de reflujo, sistema para flujo de nitrógeno (cilindro de nitrógeno 5,0, válvulas y mangueras).

\section{Equipos}

Horno MW (SAMSUNG) adaptado para síntesis química modelo AMW784S, bomba de vacío (Vacuubrand) modelo RZ 2,5, dos agitadores magnéticos (AREX), balanza analítica (Kern) modelo AB120-4, equipo de ultrasonido modelo GE 600.

\section{Síntesis del precursor $\mathrm{Cu}(\mathrm{Ac})_{2}$}

Primeramente, se calculó la masa del óxido cúprico, los volúmenes del ácido acético y anhídrido acético, utilizando un exceso de ácido acético respecto a la cantidad de moles del óxido cúprico y la relación entre el ácido acético y anhídrido acético fue (1:1). Luego, para la reacción, se armó el sistema Schlenk conectado a reflujo, los reactivos se colocaron en un balón, luego se mantuvo a agitación constante y se calentó a través de un baño de silicona a una temperatura de $150^{\circ} \mathrm{C}$ por un tiempo mayor a 8 horas bajo una atmósfera inerte. Después se filtró la solución obtenida en un sistema de filtración Schlenk que permitió la trasferencia y separación del complejo anhidro de $\mathrm{Cu}(\mathrm{Ac}) 2$ en una atmósfera inerte. A continuación, este producto fue lavado con éter etílico para arrastrar al solvente orgánico y se dejó a presión reducida por una hora, para extraer al solvente remante.
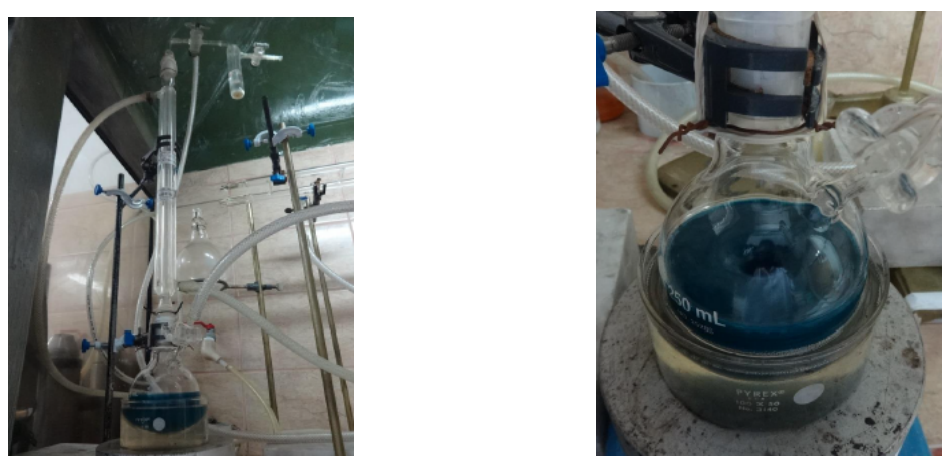

Figura 1. Imágenes del sistema Schlenk para la preparación de $\mathrm{Cu}(\mathrm{Ac})_{2}$.

\section{Síntesis de NPs Cu:}

Para un mejor diseño experimental se realizó el análisis factorial, las variables a analizar en la síntesis fueron la relación molar del $\mathrm{AA} / \mathrm{Cu}(\mathrm{Ac}) 2$ de 2,5 o 7,5, el peso molecular del PVP de $10 \mathrm{KD}$ o $40 \mathrm{KD}$ y el tipo de procedimiento $\mathrm{A}$ o $\mathrm{B}$; siendo el número de factores de tres, analizando para dos niveles por cada factor el número de experimentos es de $2^{3}$, es decir, ocho. En las tablas 1 y 2 se detallan las condiciones de estos experimentos. 
En el procedimiento A el precursor $\mathrm{Cu}(\mathrm{Ac})_{2}$ se agregó antes del calentamiento y el AA después de $3 \mathrm{~min}$. Mientras que en el procedimiento B, se agregaron de manera inversa, el AA fue el que se agregó antes y el precursor después.

\section{Procedimiento A:}

Se disolvió $0,0412 \mathrm{~g}$ de $\mathrm{Cu}(\mathrm{Ac}) 2$ en $10 \mathrm{~mL}$ de EG por ultrasonido y por separado $0,4515 \mathrm{~g}$ de PVP en 10mL de EG. Luego se procedió a mezclar las dos soluciones en un balón con agitación a $500 \mathrm{rpm}$ durante $15 \mathrm{~min}$. Por otro lado, se disolvió $0,10 \mathrm{~g}$ de AA en $10 \mathrm{~mL}$ de EG por ultrasonido y cuya relación molar con $\mathrm{Cu}(\mathrm{Ac})_{2}$ fue 2,5 (y para que la relación molar sea 7,5 se pesó $0,3 \mathrm{~g}$ ), luego se vertió a un embudo Schlenk, paralelamente se disolvió $0,12 \mathrm{~g}$ de $\mathrm{AC}$ en $10 \mathrm{~mL}$ de EG y se vertió a otro embudo Schlenk. El balón que contiene la solución del precursor metálico y PVP en EG, se colocó dentro del horno MW (figura 2) y se calentó con reflujo constante en un tiempo total de 10 min a una potencia de $10 \%$ de ciclo de irradiación. El AA se vertió gota a gota después de 3 min de haber iniciado el calentamiento y el AC después de $7 \mathrm{~min}$, a través de los respectivos embudos Schlenk, el volumen total de EG fue $50 \mathrm{~mL}$. El color rojo-pardo de la solución resultante evidenció la formación de las NPs $\mathrm{Cu}$ (figura 3).

\section{Procedimiento B:}

Es similar al procedimiento anterior, con la diferencia de que inicialmente se vertió la solución de AA y no el precursor metálico. Después de 3 min de haber iniciado el calentamiento en lugar de añadir AA se agregó el precursor metálico. Las cantidades y proporciones fueron las mismas.

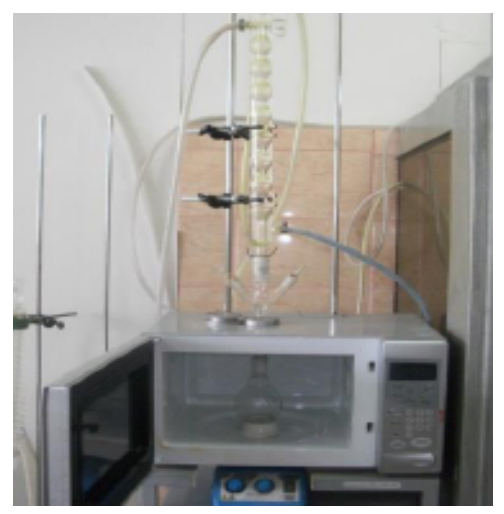

Figura 2. Microondas adaptado para síntesis química. 


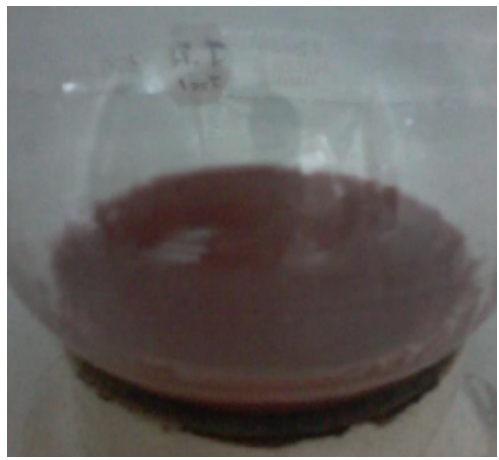

Figura 3. Solución de NPs Cu sintetizadas (color rojo-pardo).

Tabla 1. Datos de las condiciones de síntesis de las NPs $\mathrm{Cu}$.

\begin{tabular}{ccccccccc}
\hline Condiciones & A.1 & A.2 & A.3 & A.4 & B.1 & B.2 & B.3 & B.4 \\
\hline $\mathrm{Cu}(\mathrm{Ac})_{2}(\mathrm{mM})$ & 5,67 & 5,67 & 5,67 & 5,67 & 5,67 & 5,67 & 5,67 & 5,67 \\
$\mathrm{PVP}(\mathrm{mM})$ & 99,9 & 99,9 & 24,9 & 24,9 & 99,9 & 99,9 & 24,9 & 24,9 \\
$\mathrm{AA}(\mathrm{mM})$ & 14,2 & 42,6 & 14,2 & 42,6 & 14,2 & 42,6 & 14,2 & 42,6 \\
$\mathrm{AC}(\mathrm{Mm})$ & 14,3 & 14,3 & 14,3 & 14,3 & 14,3 & 14,3 & 14,3 & 14,3 \\
$\mathrm{PVP}(\mathrm{KD})$ & 10 & 10 & 40 & 40 & 10 & 10 & 40 & 40 \\
Relación molar AA/Cu$(\mathrm{Ac})_{2}$ & 2,5 & 7,5 & 2,5 & 7,5 & 2,5 & 7,5 & 2,5 & 7,5 \\
pH resultante & 4,5 & 4 & 4,5 & 4 & 5 & 4,5 & 5 & 4,5 \\
\hline
\end{tabular}

Para cada uno de los procedimientos, los ensayos 1 y 3 se diferenciaron de los ensayos 2 y 4 por la cantidad añadida de AA y por consiguiente también en la relación molar de AA/ $\mathrm{Cu}(\mathrm{Ac})_{2}$. Mientras que los ensayos 1 y 2 se diferenciaron de los ensayos 3 y 4 , en el tipo de PVP utilizado.

\section{RESULTADOS Y DISCUSIÓN}

El precursor acetato de cobre cumple un efecto estabilizante en la síntesis de las NPs $\mathrm{Cu}$, ya que los iones carboxilatos también estabilizan y se adhieren a la superficie de las NPs $\mathrm{Cu}$, teniendo un efecto similar al PVP, $\mathrm{AA}$ y $\mathrm{AC}^{2}$. Dentro de los carboxilatos cobre el más utilizado es el acetato de cobre, este puede estar de forma anhidra o hidrata, el más comercial es la forma hidratada. Sin embargo, en el mecanismo de las NPs Cu se forma la fase de óxido de cobre $\left(\mathrm{Cu}_{2} \mathrm{O}\right)$ que es un intermediario ${ }^{11}$, esta fase se puede favorecer en la síntesis si hay presencia de agua, por lo que se trabajó con $\mathrm{Cu}(\mathrm{Ac} 2)$ anhidro.

En la formación de las NPs Cu según los reactivos utilizados y los antecedentes, inicia cuando el $\mathrm{Cu}(\mathrm{Ac})_{2}$ se disuelve formando los iones $\mathrm{Ac}-\mathrm{y} \mathrm{Cu}^{+2}$, luego este último reacciona con AA 
reduciéndose primero a $\mathrm{Cu}^{+1}$ formándose nanopartículas de $\mathrm{Cu} 2 \mathrm{O}$ que tiene un color amarillo, posteriormente el AA sigue reduciendo al $\mathrm{Cu}_{2} \mathrm{O}$ formando NPs Cu metálicas ${ }^{7}$, además AA se oxida formando al ácido desoxiascórbico. Cuando hay un mayor exceso de AA se favorecerá velocidad de nucleación sobre la velocidad de crecimiento, por lo que obtendrá NPs Cu de menor tamaño9. Por otro lado, cuando se realiza la síntesis de NPs $\mathrm{Cu}$ en $\mathrm{pH}$ ácido se favorece la fase metálica de $\mathrm{Cu}$ sobre la fase $\mathrm{Cu}_{2} \mathrm{O}$.

\section{Caracterización del precursor $\mathrm{Cu}(\mathrm{Ac}) 2$ Difracción de rayos $X(D R X)$}

En la figura 4 se observa el difractograma de Rayos $\mathrm{X}$ del $\mathrm{Cu}(\mathrm{Ac})_{2}$, los picos característicos se presentan en los ángulos $2 \theta=11,24 ; 11,96 ; 14,76$ y al compararlo con los patrones estándares obtenidos de la base de datos (PDF: 27-1126), se confirmó la estructura monoclínica del $\mathrm{Cu}(\mathrm{Ac})_{2}$.

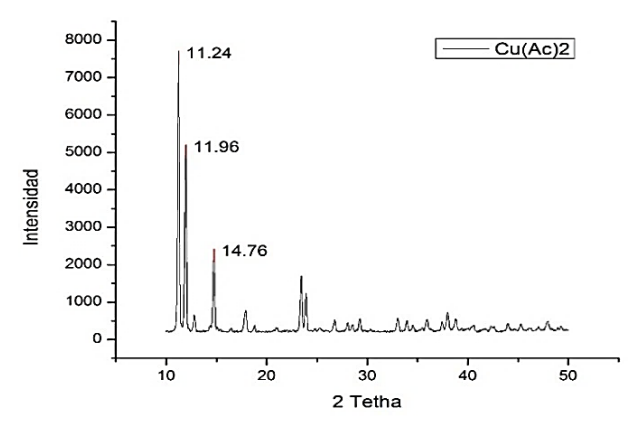

Figura 4. Difractograma del precursor $\mathrm{Cu}(\mathrm{Ac})_{2}$.

\section{Espectroscopía FTIR-ATR}

Se caracterizó al precursor $\mathrm{Cu}(\mathrm{Ac})_{2}$ mediante la técnica espectroscópica FTIR-ATR. Según Bellamy ${ }^{12}$, las bandas de absorción del grupo carbonilo $(\mathrm{C}=\mathrm{O})$ a $1.730 \mathrm{~cm}^{-1}$ y del grupo $\mathrm{C}-\mathrm{O}$ entre $1225-1268 \mathrm{~cm}^{-1}$ en las sales que provenientes de ácidos carboxílicos fuertes, estas bandas que se encuentran comúnmente en la región de los ácidos y ésteres desaparecen y son reemplazadas por otras dos bandas correspondientes al grupo $-\mathrm{COO}_{\text {as }}$ - (asimétrico) entre $1610-1550 \mathrm{~cm}^{-1} \mathrm{y}$ al $-\mathrm{COO}_{\mathrm{s}}$ - (simétrico) entre $1300-1420 \mathrm{~cm}^{-1}$. Porque se produce la resonancia de la ionización del grupo carboxilato. La presencia del grupo carboxilato del $\mathrm{Cu}(\mathrm{Ac})_{2}$ en el espectro IR (figura 5) se confirma con la presencia de las dos bandas mencionadas anteriormente. 


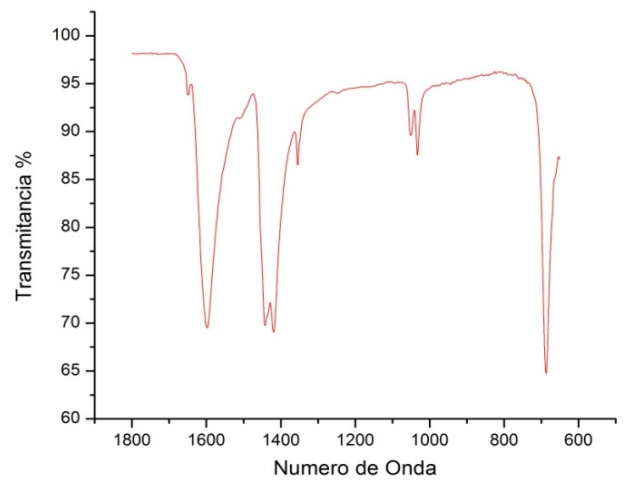

Figura 5. Espectro FTIR-ATR del precursor $\mathrm{Cu}(\mathrm{Ac})_{2}$.

\section{Caracterización de las NPs Cu Espectroscopía UV-VIS}

Los espectros UV-Vis de las NPs $\mathrm{Cu}$, sintetizadas mediante los procedimientos A y $\mathrm{B}$, se muestran en las figuras 7 y 8 , además las respectivas longitudes de onda máximas experimentales del plasmón superficial se observan en la tabla 2, esto confirma la presencia de las NPs Cu. De estos en los experimentos A1, A2, A4, B1, B2 y B4 se observa claramente el plasmón superficial de las NPs $\mathrm{Cu}$, mientras que en el experimento B3 la banda del plasmón es más ancha y en el experimento A.3 apenas se observa. Por consiguiente, las NPs Cu mencionadas en primeros experimentos tienen un menor tamaño de partícula que las síntesis A3 y B3. Esto se debe a que en estas síntesis se utilizó una relación molar de $\mathrm{AA} / \mathrm{Cu}(\mathrm{Ac})_{2}$ de 2,5 y el PVP utilizado fue 10KD. Ya que cuando la relación molar de $\mathrm{AA} / \mathrm{Cu}(\mathrm{Ac}) 2$ es mayor o igual a 5 se favorece más la reducción de los iones $\mathrm{Cu}^{+2}$ produciendo $\mathrm{NPs} \mathrm{Cu}$, favoreciendo la velocidad de nucleación sobre la velocidad de crecimiento por el método del poliol ${ }^{7,15}$. Adicionalmente, en las síntesis se utiliza el PVP de 40KD que a pesar de tener un mayor peso molecular que el PVP de 10KD, como se trabaja con el mismo peso hay cuatro veces menos PVP, por lo que el efecto dispersante será menor. Y de estos dos experimentos el de A3 está menos favorecido debido a que en el método A cuando se agrega gota a gota sobre la solución de $\mathrm{Cu}(\mathrm{Ac}) 2$ a la solución de $\mathrm{AA}$ en la reacción, al inicio el $\mathrm{Cu}(\mathrm{Ac}) 2$ a diferencia del método A en el que AA en todo momento está en exceso durante la reacción. Estos efectos explicados son sinérgicos, pero de manera desfavorables para los experimentos A3 y B3. 
Tabla 2. Longitudes de onda de las bandas de plasmón experimentales (exp.) y calculadas (cal.) y FWHM de las NPs $\mathrm{Cu}$ sintetizadas.

\begin{tabular}{ccccccccc}
\hline Condiciones & A.1 & A.2 & A.3 & A.4 & B.1 & B.2 & B.3 & B.4 \\
\hline Cu(Ac) $)_{2}(\mathrm{mM})$ & 5,67 & 5,67 & 5,67 & 5,67 & 5,67 & 5,67 & 5,67 & 5,67 \\
PVP $(\mathrm{mM})$ & 99,9 & 99,9 & 24,9 & 24,9 & 99,9 & 99,9 & 24,9 & 24,9 \\
$\mathrm{AA}(\mathrm{mM})$ & 14,2 & 42,6 & 14,2 & 42,6 & 14,2 & 42,6 & 14,2 & 42,6 \\
$\mathrm{AC}(\mathrm{Mm})$ & 14,3 & 14,3 & 14,3 & 14,3 & 14,3 & 14,3 & 14,3 & 14,3 \\
$\mathrm{PVP}(\mathrm{KD})$ & 10 & 10 & 40 & 40 & 10 & 10 & 40 & 40 \\
Relación molar AA/Cu$(\mathrm{Ac})_{2}$ & 2,5 & 7,5 & 2,5 & 7,5 & 2,5 & 7,5 & 2,5 & 7,5 \\
pH resultante & 4,5 & 4 & 4,5 & 4 & 5 & 4,5 & 5 & 4,5 \\
\hline
\end{tabular}

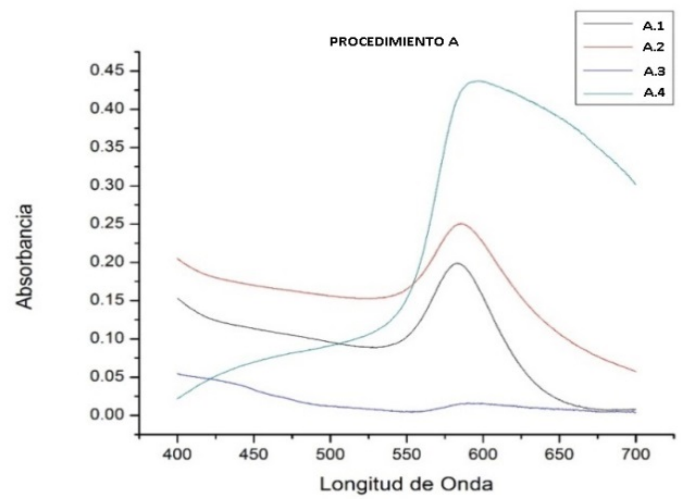

Figura 6. Espectro UV-Vis de las NPs Cu sintetizadas mediante los ensayos del procedimiento $\mathrm{A}$.

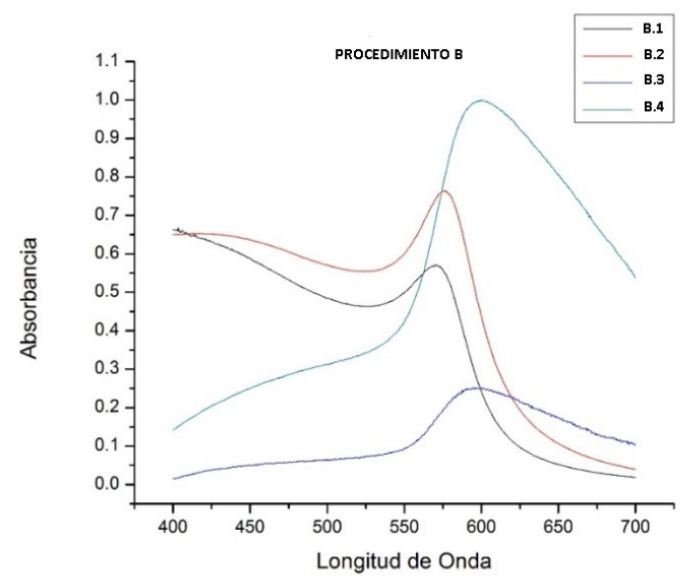

Figura 7. Espectro UV-Vis de las NPs Cu sintetizadas mediante los ensayos del procedimiento $\mathrm{A}$. 


\section{Deconvolución de los espectros UV-Vis.}

A cada uno de los espectros UV-Vis se les realizó la deconvolución en la región comprendida entre 520-640nm dentro del cual se encuentra la banda del plasmón característico de NPs $\mathrm{Cu}$. Se utilizaron funciones del tipo gaussiana que son las más apropiadas según $\operatorname{Pekian}^{14} \mathrm{y}$ estas representan las NPs Cu con tamaños similares. La función gaussiana de mayor tamaño es la principal, porque esta representa al grupo de NPs mayoritaria que hay muestra. Las longitudes de onda máxima calculadas y el ancho de banda de base media (abreviada en ingles FWHM) calculadas de las gaussianas principales, se detallan en la tabla 2. Todas las deconvoluciones se realizaron con un Chi cuadrado reducido menor a $3,00 \times 10^{-5}$, (suma de las gaussianas 1, 2 y 3). En las figuras 9 y 10 se muestran las bandas del espectro experimental de los procedimientos A.1 y B.1, respectivamente, la suma de las funciones y las funciones que conforman la gaussiana acumulada. Las deconvoluciones se realizaron con el programa Origin Pro 9.0.

Las NPs $\mathrm{Cu}$ que tienen un menor FWHM nos indica que las NPs tienen un menor tamaño de partícula ${ }^{13}$. Además, Blosi ${ }^{7}$, comprobó experimentalmente que cuando el tamaño de la NPs partícula es más pequeño, el plasmón de $\mathrm{Cu}^{0}$ tiene un desplazamiento hipsocrómico, y cuando el tamaño de la partícula es más grande tiene un desplazamiento batocrómico.

Como se observa en la tabla 2, en la longitud de banda del plasmón experimental hay un desplazamiento batocrómico de las muestras A.3 y A.4 así como en B3 y B4. Pero cuando se analizas la longitud de la banda del plasmón calculado y FWHM calculados, las muestras que tienen un mayor desplazamiento batocrómico y un mayor FWHM son las muestras A3 y B3 para los procedimientos A y procedimiento B. Por los que estas muestras de NPs Cu serían las que tiene un mayor tamaño de partícula, concordando estos resultados con el análisis cualitativo de los espectros UV-Vis de las NPs Cu en la anterior sección.

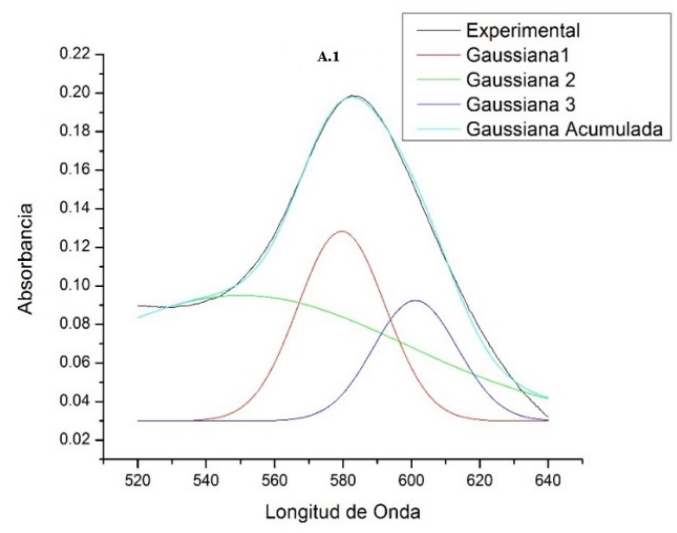

Figura 8. Deconvolución del espectro UV-Vis de NPs Cu sintetizadas por el procedimiento A.1. 


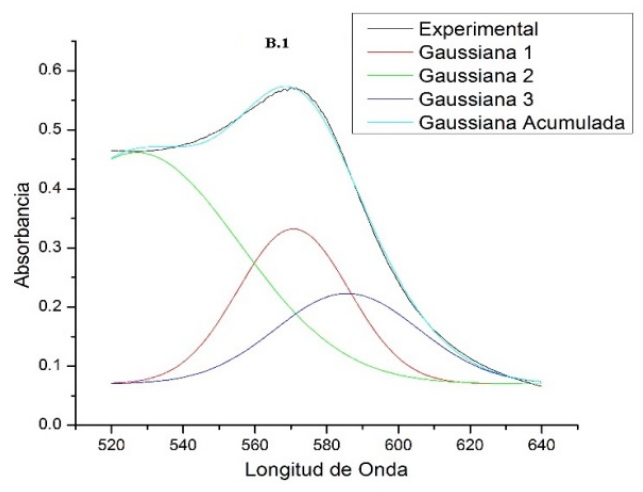

Figura 9. Deconvolución del espectro UV-Vis de NPs Cu sintetizadas por el procedimiento B.1.

\section{Análisis factorial}

En el análisis factorial se realizó para dos niveles y tres factores como se detalló en el procedimiento experimental y se evaluaron sus efectos sobre el ancho de banda de base media (FWHM) calculada y la longitud de onda máxima ( $\lambda$ máx) calculada. Los resultados de los efectos principales del análisis factorial se muestran en las figuras 10 y 11 .

En estas figuras se observa que el procedimiento B en comparación con el procedimiento A favorece la formación de NPs Cu de menor tamaño, ya que el FWHM es menor y la longitud de onda máxima tiene menores valores, es decir, hay un desplazamiento hipsocrómico. Esto es debido a que en la síntesis la solución de PVP y $\mathrm{Cu}(\mathrm{Ac}) 2$ se agrega sobre la solución de AA, por lo que el AA desde el inicio de la reacción está en exceso.

Como se mencionó anteriormente cuando se trabaja con un mimo peso el PVP de 10KD tiene un mayor efecto dispersante que el PVP de 40KD, en la figura 10 esto se demuestra en las figuras 11 y 12 donde el PVP de 10KD favorecen una menor longitud del plasmón y un menor FWHM.

En los antecedentes de síntesis tipo poliol, ${ }^{7,15}$, mencionaron que la relación molar de AA/ $\mathrm{Cu}(\mathrm{Ac})_{2}$, al ser mayor o igual a 5, favorece la formación de partículas de menor tamaño. En la figura 10 se observa que el FWHM es menor cuando la relación molar es de 7,5 en la síntesis, pero la influencian de la longitud del plasmón es casi igual para ambas relaciones molares. Por lo que la influencia en las relaciones molares no es tan significativa como en el caso de los métodos de síntesis de poliol, mediante calentamiento MW a diferencia del método del poliol por calentamiento convencional. 


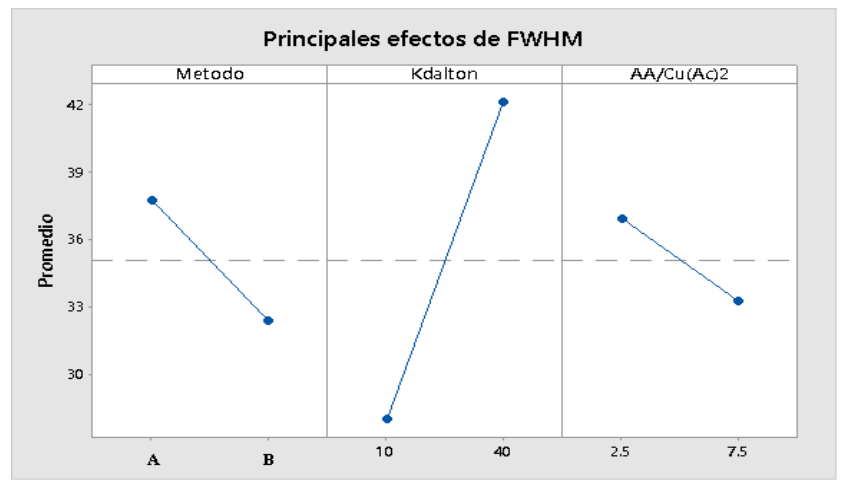

Figura 10. Diagrama de efectos principales para la variable de repuesta FWHM.

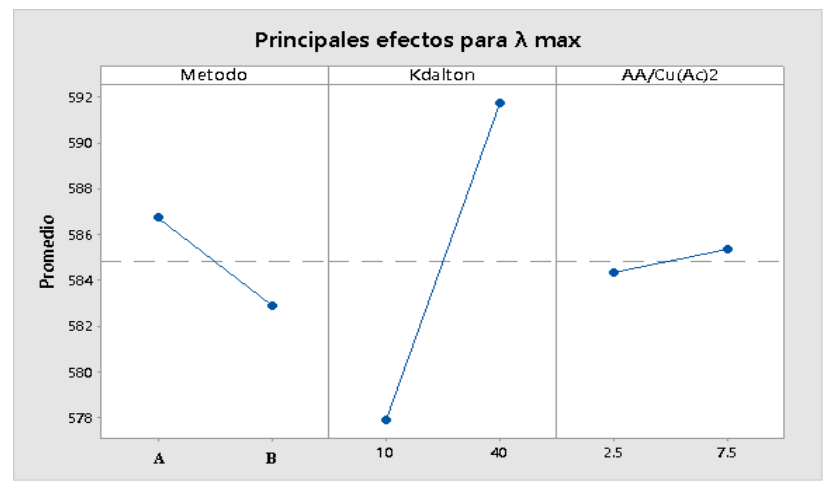

Figura 11. Diagrama de efectos principales para la variable de repuesta $\lambda$ máx.

El análisis factorial se realizó con el programa Minitab 16.

\section{Difracción de rayos X (DRX)}

En la figura 12, se observa el difractograma de las NPs $\mathrm{Cu}$ para una de las muestras sintetizadas, los picos de difracción con intensidades fuertes aparecen en ángulos de $2 \theta=$ 43,37 y 50,5; correspondientes a los planos (111) y (200) de la estructura cúbica centrada en las caras (fcc). Los dos picos de difracción concuerdan con dos de las tres señales del patrón estándar de cobre (pdf número 4-836). El tercer pico perteneciente al plano (220) no se observa, debido a la baja intensidad, siendo eliminado en el refinamiento de la línea base. Con los picos mencionados se confirmó la fase de metálica de las NPs Cu. 


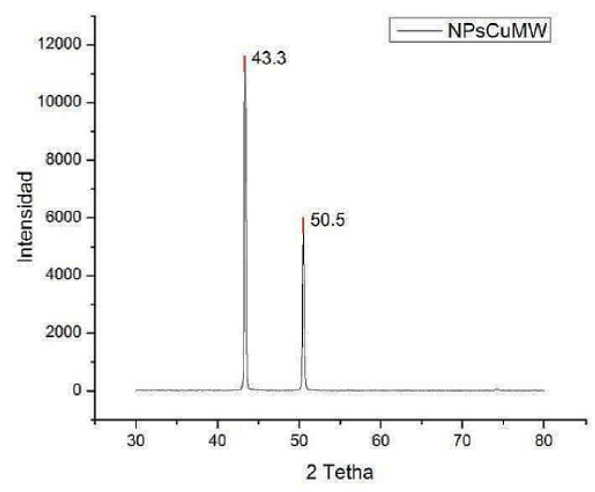

Figura 12. Difractograma de las NPs Cu sintetizadas.

El tamaño promedio de grano de las NPs Cu se calculó mediante la ecuación Debye-Scherrer y fue de 43,9nm, confirmándose la presencia de NPs Cu.

\section{Dispersión de luz dinámica (DLS)}

En la figura 13 se observa el resultado de la caracterización por DLS de una muestra de las NPs $\mathrm{Cu}$. El tamaño medio de las NPs $\mathrm{Cu}$ fue 90.9nm y de esta manera se confirmó la dimensión nanométrica de las partículas. Además, se observó dos distribuciones de NPs formadas en la muestra. El tamaño medio hallado por DLS es mayor del calculado por DRX porque considera el tamaño del radio hidrodinámico de la NPs. El PVP al adherirse a las NPs incrementa el radio hidrodinámico de las nanopartículas y por consiguiente aumenta el tamaño medio hallado por DLS.

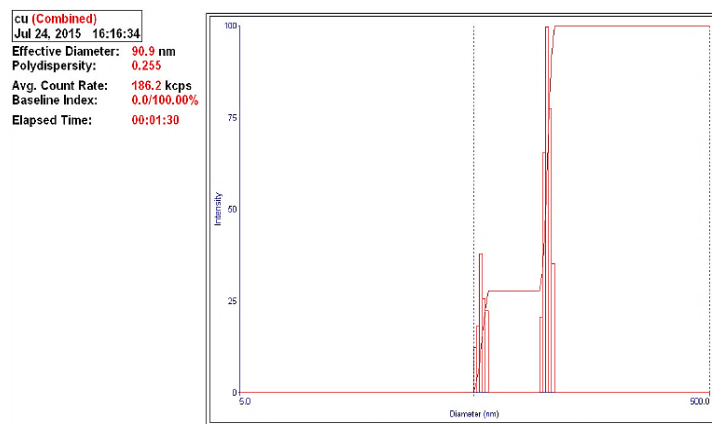

Figura 13. Imagen del resultado del software del DLS. 


\section{Microscopia electrónica de barrido (SEM)}

Las imágenes del SEM de las NPs $\mathrm{Cu}$ se observan en la figura 14, ambas imágenes pertenecen a la misma muestra con diferentes aumentos, se observa algunas de mayor y otras de menor tamaño, debido a que algunas NPs $\mathrm{Cu}$ se han aglomerado formando NPs secundarias más grande, esto se debe a que en la medición de la muestra con el equipo SEM fueron realizada tres meses después de la síntesis, dando tiempo a un mayor efecto del fenómeno de coalescencia. La morfología de estas partículas es diversa. La medición de la muestra se realizó en el solvente original, donde las nanopartículas están en una suspensión de etilenglicol, por lo que se utilizó el modo ambiental, no se pudo realizar mediciones a mayores aumentos, ya que en el modo ambiental no se ejerce una mayor presión de vacío, ya que los electrones necesitan un alto vacío para mejorar la resolución.
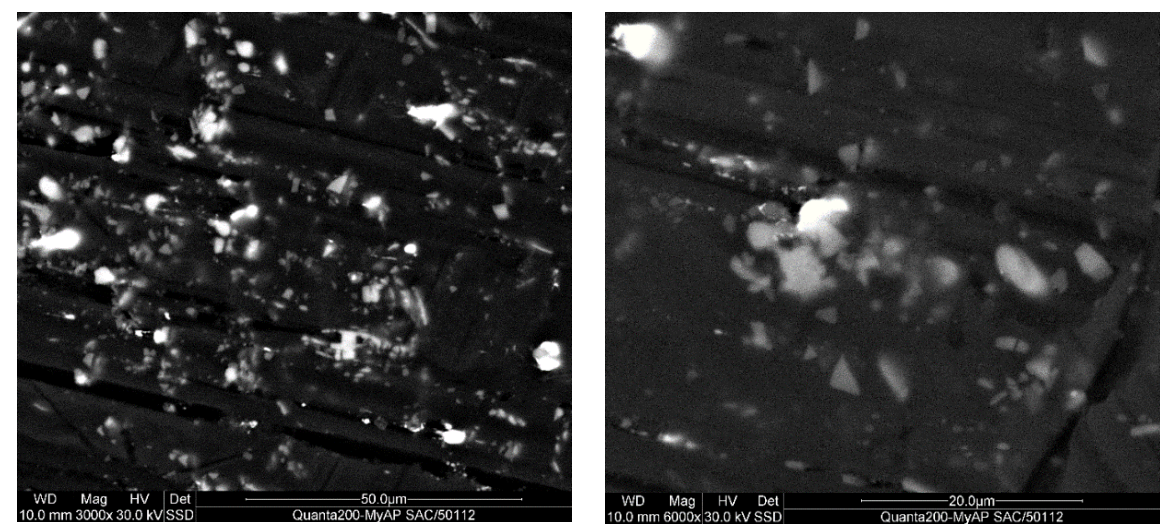

Figura 14. Imagenes SEM de las NPsCu: a 3000 de aumento (izquierda), a 600 de aumento (derecha).

\section{CONCLUSIONES}

Los resultados indicaron que se logró sintetizar NPs $\mathrm{Cu}$ con los métodos mostrados, observando el plasmón característico en el espectro UV-Vis. El resultado del tamaño de grano por DRX, de una de las muestras, fue de $44 \mathrm{~nm}$ y el tamaño del radio hidrodinámico por DLS fue de $90.9 \mathrm{~nm}$ confirmando la presencia de NPs Cu. En la evaluación de los dos procedimientos de síntesis (A y B), en ambos se obtuvieron NPs $\mathrm{Cu}$, pero el procedimiento $\mathrm{B}$ fue mejor que el procedimiento A pues las NPs $\mathrm{Cu}$ sintetizadas tuvieron menor tamaño, lo cual fue demostrado mediante la deconvolución de los espectros UV-Vis y el análisis factorial. Con el mismo criterio también se determinó que para un mismo peso de PVP las síntesis de nanopartículas de cobre se favorecen cuando el peso molecular del PVP es menor que fue de $10 \mathrm{KD}$. 


\section{AGRADECIMIENTOS}

Se agradece al FINCyT por todo el financiamiento brindado en el desarrollo de esta investigación mediante el proyecto No 135-FINCyT-IB-2013. A la Universidad Nacional Mayor de San Marcos por ser una gran institución y brindarnos la oportunidad de pertenecer a ella.

\section{REFERENCIAS BIBLIOGRÁFICAS}

1. Barrabés N, Just J, Dafinov A, Medina F, Fierro JLG, Sueiras JE, et al. Catalytic Reduction of Nitrate on Pt-Cu and Pd-Cu on Active Carbon using Continuous Reactor: The Effect of Copper Nanoparticles. Appl Catal B-Environ. 2006; 62: 77-85.

2. Eastman JA, Choi SU, Li S, Yu W, Thompson LJ. Anomalously Increased Effective Thermal Conductivities of Ethylene Glycol-Based Nanofluids Containing Copper Nanoparticles. Appl Phys Lett. 2001; 78(6): 718-720.

3. Hoover NN, Auten BJ, Chandler BD. Tuning Supported Catalyst Reactivity with Dendrimer-Templated Pt-Cu Nanoparticles. J Phys Chem B. 2006; 110(17): 8606-8612.

4. Yu W, Xie H, Chen L, Li Y, Zhang C. Synthesis and Characterization of Monodispersed Copper Colloids in Polar Solvents. Nanoscale Res Lett. 2009; 4(5): 465-470.

5. Zhang H, Siegert U, Liu R, Cai WB. Facile Fabrication of Ultrafine Copper Nanoparticles in Organic Solvent. Nanoscale Res Lett. 2009; 4(7): 705-708.

6. Chung SC, Yun HJ, Min GK, Hyuck ML. Control of chemical kinetics for sub-10 nm Cu nanoparticles to fabricate highly conductive ink below $150^{\circ} \mathrm{C}$. Nanotechnol. 2012;23(6): 5601-5609.

7. Blosi M, Albonetti S, Dondi M, Martelli C, Baldi G. Microwave-assisted polyol synthesis of Cu nanoparticles. J Nanopart Res. 2011; 13(1): 127-138.

8. Kawasaki H, Kosaka Y, Myoujin Y. Microwave-assisted polyolsynthesis of copper nanocrystals without using additional protective agents. Chem Commun. 2011; 47: $7740-7742$

9. Nikkam N, Ghanbarpour M, Saleemi M, Haghighi EB, Khodabandeh R, Muhammeda $\mathrm{M}$, et al. Experimental investigation on thermo-physical properties of copper/diethylene glycol nanofluids fabricated via microwave-assisted route. Appl Therm Eng. 2014; 65: 158-165.

10. Nishioka M, Miyakawa M, Daino Y, Kataoka H, Koda H, Sato K, et al. Single-Mode Microwave Reactor Used for Continuous Flow Reactions under Elevated Pressure. Ind Eng Chem Res. 2013; 52(12): 4683-4687.

11. Raspolli Galletti AM, Antonelli C, Marracci M, Piccinelli F, Tellini B. Novel microwave-synthesis of $\mathrm{Cu}$ nanoparticles in the absence of any stabilizing agent and their antibacterial and antistatic applications. Appl Surf Sci. 2013; 280: 610-618.

12. Bellamy L. The Infra-Red Spectra of Complex Molecules. New York: Springer Netherlands; 1975. 
13. Garcia Rey N. Interaction of a Copper Surface with Light: Plasmons, Electrons \& Molecular Vibrations. [Tesis doctoral]. Liverpool, Reino Unido: University of Liverpool; 2011.

14. Pelikan P, Cepan M, Liska M. Application of numerical Methods in Molecular Spectroscopy. Boca Raton, Florida: CRC Press; 1993

15. Yu W, Xie H, Chen L, Li Y, Zhang C. Synthesis and Characterization of Monodispersed Copper Colloids in Polar Solvents. Nanoscale Res Lett. 2009; 4(5): 465-470. 